\title{
Les systèmes de répartition des élèves au prisme de la justice sociale en éducation : deux études de cas en Suisse romande
}

\author{
Sonia Revaz, Université de Genève \\ Kilian Winz, Université de Genève
}

DOI : 10.51186/journals/ed.2021.11-1.e466

\section{Résumé}

Le concept de justice sociale constitue aujourd'hui un référentiel partagé, du moins lorsqu'il est appliqué à la sphère de l'école. Pourtant, les conclusions dominantes des travaux de recherche en éducation sur la justice sociale, qui valorisent l'équité des systèmes éducatifs, coïncident peu avec les représentations et les pratiques des personnes impliquées dans l'élaboration des lois scolaires et leur mise en œuvre en classe. Partant de ce constat, cet article mobilise les outils de la sociologie de l'éducation et des sciences politiques pour éclairer la façon dont les membres du Parlement et les enseignant-es définissent et interprètent la justice sociale à l'école. II s'appuie sur deux études de cas - les réformes de l'enseignement secondaire I dans les cantons de Neuchâtel et de Vaud - construites sur l'analyse de débats parlementaires et d'entretiens semi-directifs réalisés auprès de professionnel-les de l'école. Articulées, les études de cas montrent que les député-es parlementaires et les professionnel-les ont des représentations de la justice sociale fondées non seulement sur leurs idées et intérêts, mais également sur des schèmes institutionnels locaux. Nous verrons que ces représentations sont au cœur de l'action publique éducative puisqu'elles créent une dissonance entre les intentions politiques et leur transposition en classe.

Mots-clés : justice sociale, politiques éducatives, réforme scolaire structurelle, représentations, Suisse romande

\section{Abstract}

The concept of social justice is today a shared frame of reference, at least when applied to the school domain. However, the dominant conclusions of educational research on social justice, which value the equity of educational systems, do not coincide with the representations and practices of those involved in the development of school laws and their implementation in the classroom. Based on this observation, we utilize the tools of sociology of education and political science analyze the way members of parliament and teachers 
define and interpret social justice in schools. It is based on two case studies - the reforms of lower secondary education in the cantons of Neuchâtel and Neuchâtel - built on the analysis of parliamentary debates and semi-structured interviews with school professionals. The case studies show that both parliamentarians and professionals have representations of social justice based not only on their own ideas and interests but also on local institutional schemes. We will see that these representations are at the heart of public action in education since they create a dissonance between political intentions and their transposition into the classroom.

Keywords: educational policies, French-speaking Switzerland, representations, social justice, structural reform

\section{INTRODUCTION}

En Suisse romande, comme dans d'autres régions et pays, la volonté de lutter contre les inégalités à l'école s'est manifestée durant la deuxième moitié du 20ème siècle. À Genève, par exemple, à la fin des années 1970, la Loi sur l'instruction publique introduit la notion de lutte contre les inégalités en déclarant que l'école « doit tendre à corriger les inégalités de chances et de réussite scolaire des élèves dès les premières années de l'école » (art. 10). La concrétisation de cette lutte dans des modifications des systèmes éducatifs est cependant plus tardive en Suisse romande que dans d'autres pays comme la France ${ }^{1}$ où les premiers dispositifs d'éducation prioritaire et d'école inclusive se sont développés dès les années 1980. Ce n'est en effet qu'au début des années 2000 que des transformations législatives sont apportées aux systèmes romands dans le but de réduire les inégalités à l'école². Ces modifications prennent des formes variables puisqu'elles ciblent différents types d'inégalités et se basent sur différentes définitions de ce qui est le plus juste pour les élèves.

Si, dans le canton de Genève, la lutte contre les inégalités s'est surtout traduite par l'instauration d'un réseau d'enseignement prioritaire inspiré notamment du modèle français, dans d'autres cantons, elle s'est réalisée à travers la révision des modèles de répartition des élèves dans l'enseignement secondaire ; l'idée étant de réduire les pratiques de sélection qui tendent à accentuer les inégalités de départ. C'est ce qu'il s'est passé dans les cantons romands de Vaud et Neuchâtel en 2013 et 2015, suite à l'impulsion d'acteurs/trices politiques. Avant les réformes, dans les deux cantons, les élèves étaient réparti-es dans trois filières de niveaux d'exigences différents sur la base de leurs résultats de fin de primaire. Les deux systèmes se caractérisaient par ailleurs par de faibles possibilités de réorientation. Les

\footnotetext{
${ }^{1}$ Les politiques d'éducation prioritaire naissent en France dans les années 1960 dans le but de réduire l'impact des inégalités socioéconomiques sur la réussite scolaire des élèves en renforçant l'action éducative dans les zones les plus touchées par l'échec scolaire.

2 Ici, on ne prend pas en compte les "classes spéciales " créées au début du XIXe siècle pour les enfants porteurs/euses de handicap, qualifiés alors d' « arriéré-es » (Avvanzino, 1993).
} 
deux cantons ont toutefois opéré des modifications différentes. Dans le canton de Vaud, l'une des trois filières - la moins exigeante - a été supprimée et la perméabilité entre les deux restantes a été renforcée. À Neuchâtel, le système filiarisé a été abandonné au profit de classes hétérogènes avec, pour certaines disciplines, des groupes de niveaux.

Ces modifications des systèmes scolaires neuchâtelois et vaudois ont en commun une dimension pratique et une dimension idéologique : la restructuration du système éducatif et la lutte contre les inégalités. Si ces deux dimensions, en plus d'une étroite proximité géographique, constituent le point de départ du rapprochement que nous proposons entre les deux réformes, ce sont également leurs singularités qui invitent à les mettre en parallèle. La comparaison est, en effet, particulièrement riche en cela qu'elle montre comment le sens des réformes et, a fortiori, de la justice sociale à l'école, est déterminé par des processus vernaculaires, c'est-à-dire propres à chaque contexte local.

Ces deux dimensions, pratique et idéologique, sont inévitablement intriquées, puisqu'elles concernent la gestion de l'hétérogénéité des niveaux des élèves, c'est-à-dire des inégalités de départ. Or, depuis plusieurs décennies, la recherche montre que les inégalités scolaires sont fortement liées aux inégalités sociales, et ce, à l'échelle internationale (Bourdieu \& Passeron, 1964 ; Coleman, et al., 1966 ; Felouzis, 2020 ; Monseur \& Crahay, 2008). Becker (2013) l'a aussi montré en Suisse : les parcours et les résultats des élèves diffèrent selon la catégorie socioprofessionnelle de leur famille. Raisonner sur ce qui est le plus juste, du point de vue de la structure du système éducatif, pour les élèves de niveaux scolaires différents revient, par conséquent, aussi à raisonner sur ce qui est le plus juste pour les élèves issu-es de milieux socioéconomiques différents.

La question de la structure du système éducatif constitue un enjeu politique majeur, car il en va de l'intégration des jeunes générations dans la société et donc de la participation de l'école à la justice sociale, puisque celle-ci renvoie à une participation de tous les individus à la vie sociale (Fraser, 2004). Et si les politiques publiques sont souvent interprétées diversement par les acteurs/trices - autant celles/ceux qui les élaborent que celles/ceux chargé-es de les mettre en œuvre (Palier \& Surel, 2005), on peut se demander comment les individus qui ont participé à ces réformes définissent et conçoivent la justice sociale à l'école. Nous proposons, dès lors, d'analyser la façon dont les représentations des acteurs/trices politiques et professionnel-les déterminent les processus d'élaboration et de mise en œuvre de politiques de justice sociale à l'école. En d'autres termes, il s'agit de mettre en évidence le poids des cadres cognitifs et normatifs sur le sens et la forme que prend la justice sociale à l'école. Une telle entreprise exige l'observation de discours et de pratiques relatifs aux deux 
paradigmes $^{3}$ de la justice sociale : la redistribution économique et la reconnaissance culturelle (Dubet, 2014a ; Fraser, 2004 ; Lynch \& Payet, 2011). Alors que le premier vise l'égalité sociale à travers la lutte contre les inégalités socioéconomiques notamment, le second revendique la reconnaissance sociale de toutes les identités. Pour répondre à cette ambition et comprendre la façon dont la justice sociale est pensée par les acteurs/trices chargé-es de l'interpréter dans les politiques et les pratiques éducatives, deux études de cas sur les réformes structurelles des cantons de Vaud et de Neuchâtel sont mobilisées.

\section{QUELLES FORMES PREND LA JUSTICE SOCIALE À L’ÉCOLE ?}

Rendre compte des façons dont les acteurs/trices politiques et les professionnels/les de l'enseignement appréhendent, définissent et traduisent le concept de justice sociale à l'école, nécessite la mobilisation de deux cadres théoriques complémentaires : un premier relatif à la sociologie des systèmes éducatifs qui octroie des outils pour appréhender la justice sociale au cœur des modèles de répartition des élèves et un second qui renferme davantage une sociologie générale des acteurs/trices, tant politiques que « de terrain ». Celui-ci permet d'éclairer la façon dont l'ensemble des acteurs/trices d'un système contribue à sa construction.

\subsection{Les modèles de gestion des inégalités de départ : des possibilités multiples}

Tous/tes les élèves n'entrent pas égales/aux à l'école : " II est évident que tous les membres de la société ne sont pas égaux en termes matériels et symboliques, et que les élèves ne sont donc pas tous égaux de fait » (Friant, 2013, p. 138). On sait toutefois que les inégalités scolaires ne découlent pas uniquement des caractéristiques individuelles des élèves ; l'école y joue également un rôle à travers son système de distribution des ressources. Or, le choix du traitement des élèves repose sur des valeurs liées à la justice (Dubet, 2004 ; Friant, 2012 ; Meuret, 1999 ; Monseur \& Demeuse, 2001). Quel type d'inégalités est admis ou non - les inégalités de traitement ou les inégalités de résultats ? Ce choix se matérialise souvent à travers différentes façons de répartir les élèves à l'école (Monseur \& Demeuse, 2001). À partir de systèmes éducatifs européens distincts, Mons (2007) propose une typologie qui distingue quatre modèles d'attribution des élèves : la séparation, l'intégration individualisée, l'intégration à la carte et l'intégration uniforme. Le premier correspond à un modèle hiérarchique et peu individualisé dans lequel les élèves sont classé-es et réparti-es en fonction de leur niveau de compétences. Le second constitue, à peu de choses près, son opposé : il repose sur un " mélange » d'élèves de différents niveaux dans des classes dans lesquelles l'enseignement différencié est fortement pratiqué. Le troisième modèle est

\footnotetext{
3 Dans ses travaux plus récents, Fraser (2010) ajoute une troisième dimension : la représentation politique. Cet ajout est inspiré de la distinction opérée par Max Weber dans Économie et Société (1922) entre trois modes de stratification : la classe, le statut et l'ordre politique. Selon l'auteure, les rapports de représentation politique constituent des problèmes de justice du fait que tous les individus ne sont pas également représentés politiquement dans la vie sociale.
} 
construit sur le même fonctionnement ; il sépare néanmoins les élèves dans des classes de niveaux différents pour les enseignements de certaines disciplines. Enfin, le quatrième modèle renonce au regroupement des élèves en fonction de leur niveau et gère les inégalités de départ au moyen de redoublements. Pour chaque système éducatif, le choix du modèle de répartition est déterminant dans la mesure où les différentes structures « ne génèrent pas [...] les mêmes niveaux d'inégalités entre élèves » (Felouzis \& Goastellec, 2015, p. 6).

Malgré la coexistence de ces modèles et de ces deux logiques de justice à l'école, il existe aujourd'hui une tendance internationale qui définit l'équité comme une valeur centrale en éducation (Dupriez \& Verhoeven, 2006). Cette tendance est attestée par différents travaux de sociologie et de sciences de l'éducation qui montrent que cette équité n'est pas une réalité tangible dans de nombreux pays (Dumay \& Dupriez, 2009 ; Duru-Bellat, et al., 2004 ; Felouzis \& Goastellec, 2015). Dès lors, on peut se demander si cela provient du décalage entre ce que dit la recherche en éducation sur la justice sociale - à savoir que l'équité est une valeur phare pour l'école - et les conceptions vernaculaires des acteurs/trices politiques et des acteurs/trices de terrain. Ce décalage s'observe en effet lorsque l'on met en perspective le discours scientifique dominant et celui - ou ceux - formulé par les acteurs/trices impliquées dans l'élaboration des lois et dans leur mise en œuvre dans les classes.

\subsection{Des choix politiques et professionnels construits sur des schèmes cognitifs et normatifs}

Si la justice sociale prend une signification différente lorsqu'elle est objectivée en politique éducative, c'est parce que les processus politiques sous-jacents sont producteurs de sens. En d'autres termes, l'action publique en éducation repose largement sur les cadres cognitifs et normatifs des acteurs/trices qui y participent (Muller \& Surel, 1998). En effet, « la promotion d'un problème [est inséparable] des processus cognitifs et normatifs de définition et de qualification (ou de "problématisation ") qui donnent sens à ce problème et conditionnent les termes des débats, du mode de traitement et des éventuelles décisions » (Garraud, 2010, p. 58). Dès lors, autant les acteurs/trices politiques responsables de l'élaboration de la loi que les professionnels/les responsables de la mettre en œuvre sur le terrain donnent du sens à la problématique soulevée.

Par ailleurs, l'intérêt de mettre en perspective l'interprétation que les acteurs/trices qui élaborent la réforme ont de la justice sociale et l'interprétation qu'en ont celles/ceux qui la mettent en œuvre repose sur le rejet d'une approche fonctionnaliste de l'implémentation d'une politique : «Mettre en œuvre, c'est interpréter » (Lessard \& Carpentier, 2015, p. 189). Dès lors, bien que les acteurs/trices politiques donnent un sens à la justice sociale, les acteurs/trices de terrain - les street-level bureaucrats (Lipsky, 2010) - détiennent un pouvoir discrétionnaire majeur leur permettant de réinterpréter ce sens, de le contourner, voire de le réorienter en fonction de leurs représentations et intérêts. Cette appropriation des injonctions 
d'une réforme est, par ailleurs, d'autant plus puissante lorsque les enseignant-es appréhendent leur profession dans une logique collective. Regroupés en sous-groupes, les membres du corps enseignant peuvent faire front face aux injonctions et donner un nouveau sens à une réforme : " the nature and structure of formal networks and informal alliances among teachers play a powerful role in shaping the sensemaking process and ultimately the kind of sense that is made » (Coburn, 2004, p. 145). Animés par des représentations et des intérêts communs - l'idéologie professionnelle (Lessard, et al., 2008b), les « class-level bureaucrats ", pour reprendre la pensée terminologique de Lipsky, peuvent ainsi retraduire le sens d'une politique.

\section{DEUX RÉFORMES IMPULSÉES POUR RÉDUIRE LES INÉGALITÉS SCOLAIRES}

Les deux réformes étudiées concernent la dernière étape de la scolarité obligatoire ${ }^{4}$ en Suisse : l'enseignement secondaire I. Dans le canton de Vaud, le processus de réforme ${ }^{5}$ est entamé lorsque deux groupements de député/es sollicitent le Gouvernement (le Département de la formation, de la jeunesse et de la culture - DFJC) ${ }^{6}$ pour entreprendre des modifications de la structure du secondaire I. La requête découle d'un double constat : celui de l'inefficacité du système - en termes de résultats et de réussites scolaires - et du rôle des facteurs structurels sur les inégalités entre élèves. Le défi est conséquent : il s'agit d'améliorer l'efficacité de l'enseignement secondaire I en tenant compte des inégalités de départ. L'option envisagée repose sur la révision du modèle de répartition des élèves, qui consiste en trois filières aux exigences différentes : "voie secondaire de baccalauréat (VSG) »; « voie secondaire générale (VSG) » et « voie secondaire à options (VSO) »7. Deux projets de loi s'affrontent. Le premier est porté par le Département, animé notamment par les interpellations des deux groupes parlementaires, et le second est une initiative populaire ${ }^{8}$

\footnotetext{
${ }^{4}$ La Suisse est un État fédéral. La responsabilité de la scolarité obligatoire relève donc principalement de la compétence des cantons. La scolarité obligatoire dure onze ans et regroupe les degrés primaire et secondaire I. L'enseignement primaire est organisé en classes hétérogènes dans tous les cantons. En revanche, la structure de l'enseignement secondaire I varie entre les cantons. La tendance est plutôt d'opter pour un système mixte articulant des filières (le nombre et les intitulés varient entre les cantons) pour l'enseignement des disciplines principales (généralement la langue 1, la langue 2 et les mathématiques) et des classes hétérogènes pour l'enseignement des autres disciplines.

${ }^{5} \mathrm{La}$ réforme vaudoise concerne l'entièreté de la scolarité obligatoire. Notre intérêt ici se focalise toutefois essentiellement sur les modifications apportées à l'enseignement secondaire I.

6 En Suisse, le Gouvernement est composé de différents Départements (équivalents aux Ministères) dans lesquelles sont réparties les responsabilités des différents secteurs publics. Leurs noms varient entre les cantons et peuvent changer d'une année à l'autre, suite à la répartition des Départements aux différents Chefs (Ministres). Par exemple, alors que le Département responsable des questions liées à l'éducation s'intitule Département de la formation, de la jeunesse et de la culture dans le canton de Vaud, on parle de Département de l'instruction publique dans le canton de Genève et de Département de l'économie et de la formation dans le canton du Valais.

7 De la plus exigeante à la moins exigeante.

8 Le système de démocratie directe participative en Suisse donne la possibilité à la population de participer aux décisions politiques. L'initiative populaire est un outils démocratique qui permet aux citoyens suisses de proposer la modification d'une loi ou un projet de loi.
} 
(“École 2010 : sauver l'école ») rédigée par trois associations ${ }^{9}$ d'enseignant-es et de parents d'élèves. Le projet du Département plaide pour une réduction des filières (par le biais de la suppression de la VSO, jugée trop stigmatisante pour les élèves faibles) afin de rendre l'enseignement plus hétérogène et d'atténuer les inégalités entre élèves. Dans une optique idéelle opposée, l'initiative populaire défend le maintien des trois filières jugées indispensables, car à l'image de la réalité des inégalités entre élèves d'une part, et de la réalité du marché du travail d'autre part. Les deux projets de loi sont soumis au vote populaire en 2011 et c'est le projet du Département qui l'emporte ; il entre en vigueur en 2013.

Dans le canton de Neuchâtel, les prémices de la réforme sont similaires. Elle naît en 2010 d'une démarche du parti socialiste, réclamant du Parlement une intervention « pour un nouveau modèle du secondaire I ". Celle-ci vise particulièrement le système de répartition des élèves alors en vigueur, caractérisé, comme dans le canton de Vaud, par un modèle de séparation (Mons, 2007) dans lequel les élèves sont affecté-es dans des sections aux exigences variables en fonction de leurs résultats en fin de primaire. Les signataires dénoncent le caractère arbitraire de la répartition des élèves et l'accusent d'occasionner de lourdes conséquences sur l'avenir de ces derniers. Pour y pallier, le Gouvernement (Département de l'éducation et de la famille) élabore un projet de loi qui poursuit deux objectifs : valoriser les élèves de toutes les sections et renforcer les compétences de l'ensemble des élèves. Concrètement, il propose d'introduire un nouveau mode de répartition des élèves qui s'articule comme un système d'intégration à la carte (Mons, 2007) : les élèves sont intégré-es dans des classes indépendamment de leur niveau scolaire et, pour les disciplines principales, elles/ils sont séparé-es en deux niveaux (le niveau 1 à exigences faibles et le niveau 2 à exigences élevées). Le projet est accepté au Parlement ${ }^{10}$ par une écrasante majorité des député/es (97 voix favorables contre une) et entre en vigueur en 2015.

\section{ANALYSER LE POIDS DES CADRES COGNITIFS ET NORMATIFS SUR LE SENS ET LA FORME DE LA JUSTICE SOCIALE À L'ÉCOLE}

L'analyse du décalage entre ce que dit la recherche en éducation sur la justice sociale à l'école et les représentations qu'en ont les acteurs/trices qui doivent s'y confronter dans des politiques et des pratiques éducatives se fait à partir de deux matériaux. Le cas vaudois donne à voir la façon dont le paradigme de justice sociale est interprété en termes politiques par les acteurs/trices responsables de la rédaction du texte de loi. Dès lors, on ne peut se dispenser d'une approche cognitive de la réforme puisque celle-ci permet d'appréhender le

\footnotetext{
9 Les trois associations sont les suivantes : l'association d'enseignant-es AVEC (Association vaudoise pour une école crédible) et deux associations de parents d'élèves : l'AVPC (Association vaudoise des parents chrétiens) et l'ASPICS (Association des parents intéressés et concernés par la scolarité).

10 Le vote populaire intervient automatiquement en cas d'initiative populaire. En revanche, si une modification de loi ou nouvelle loi est proposée par le Parlement, le vote n'intervient pas obligatoirement. Le peuple a, toutefois, la possibilité de s'opposer à un changement initié par le Parlement en déposant un référendum exigeant le passage par le vote populaire.
} 
discours comme un indicateur des cadres cognitifs et normatifs à la source d'une politique (Muller, 2000 ; Surel, 2000). L'approche cognitive s'est concrétisée au travers d'analyses des transcriptions des séances parlementaires, auparavant réunies dans un même corpus ${ }^{11}$. Deux méthodes d'analyse ont été articulées pour examiner les interprétations de la justice sociale dans la réforme vaudoise : des analyses thématiques dites «manuelles » (Paindorge, et al., 2015) et des analyses lexicales à l'aide du logiciel Alceste, dont la fonctionnalité principale est la lexicométrie.

Le cas neuchâtelois vise, ensuite, à rendre compte de la façon dont les professionnel-les interprètent la justice sociale dans l'exercice de leur profession. Le propos s'ancre sur une soixantaine d'entretiens semi-directifs de 60 minutes réalisés entre janvier et juin 201912 auprès d'enseignants et enseignantes exerçant au sein de cinq établissements ${ }^{13}$ sélectionnés sur le territoire cantonal. Bien que des entretiens ont été menés avec la majorité des acteurs/trices de l'éducation comme des politiques, membres du service cantonal de l'enseignement obligatoire, syndicats ou encore directions d'établissements, nous mobilisons ici principalement les analyses des entretiens menés avec le corps enseignant ${ }^{14}$.

La méthode de l'entretien est particulièrement adaptée pour la recherche sur les représentations détaillées des acteurs/trices sociaux. Ce dispositif permet notamment de faire dialoguer les histoires des individus avec leurs intérêts et leurs idées. Comme le mentionnait Van der Maren (1996), cette méthode « vise à obtenir des informations sur les perceptions, les états affectifs, les jugements, les opinions, les représentations des individus à partir de leur cadre personnel de référence et par rapport à des situations actuelles » ( $p$. 312). Les entretiens ont fait l'objet d'analyses thématiques effectuées avec le logiciel AtlasTi afin de mettre au jour la façon dont les enseignant-es perçoivent la justice sociale relativement aux fonctions qu'elles/ils attribuent à l'école et, plus précisément, au secondaire I.

\section{INTERPRÉTATIONS ET TRADUCTIONS DE LA JUSTICE SOCIALE DANS LES SPHĖRES POLITIQUES ET PROFESSIONNELLES : DEUX ÉTUDES DE CAS}

\subsection{Représentations idéelles et traductions institutionnelles ambivalentes de la justice sociale dans le canton de Vaud}

L'analyse des débats tenus par les acteurs/trices politiques vaudois-es est particulièrement riche pour rendre compte de la façon dont les questions de ce qui est le plus juste pour les élèves du secondaire I ont été négociées. Elle témoigne particulièrement des conflits cognitifs

\footnotetext{
11 Le corpus compte 145 pages (100 281 mots/629 545 signes).

12 Le nouveau système est entré en vigueur en août 2015.

13 Les établissements présentent des profils socioéconomiques variés.

14 Nous mobilisons également une situation discutée avec une direction d'établissement qui permet l'explicitation des propos théoriques.
} 
autour de la notion d'égalité des chances. Celle-ci se discute autour d'une question : quelle structure scolaire est la plus juste du point de vue des apprentissages des élèves ? La question se cristallise au travers de propositions concrètes relatives au degré d'hétérogénéité des classes.

Quand bien même les modèles structurels défendus sont différents, un argument central est commun aux deux camps ${ }^{15}$ : le soutien aux élèves en difficulté (Revaz, 2020). L'argument se décline en deux dimensions : leur réussite scolaire et leur expérience scolaire. Celles-ci sont en effet évoquées par l'ensemble des député-es, quel que soit le modèle soutenu. Pour les député-es en faveur de la réduction des filières et de l'instauration de classes hétérogènes, la modification de la loi est indispensable pour les élèves en difficulté, car celles/ceux-ci sont sujet-tes à de fortes stigmatisations. Selon ces député-es, l'aspect cloisonné du système vaudois induit la catégorisation des élèves (à partir des étiquettes : "Les VSB ", "les VSG » et « les VSO ») et la mauvaise réputation de la VSO est source de stigmate : « [...] Nous nous enferrons dans une école élitiste, qui stigmatise celles et ceux qui sont dans les filières " dépotoir » - vous me permettrez cette qualification qui vient de ceux qui y sont euxmêmes » (Extrait d'une prise de parole d'un député en faveur des classes hétérogènes). Elles/ils estiment que la popularité pernicieuse de la VSO nuit aux perspectives professionnelles des élèves qui y sont inscrit-es, du fait que les employeurs/euses auraient tendance à moins les embaucher, au profit des élèves issu-es des deux autres filières. Quant aux député-es partisan-es du maintien du système à trois voies, elles/ils estiment que les élèves faibles doivent être séparé-es des autres pour deux raisons. D'une part, pour bénéficier d'un enseignement adapté à leur niveau et compenser leurs difficultés scolaires. D'autre part, pour éviter un nivellement par le bas des classes : « Si l'école doit donner les mêmes chances à tous, elle ne peut mélanger les élèves dans un système excessivement inclusif, qui découragerait les plus faibles et freinerait les plus forts » (Extrait d'une prise de parole d'un député en faveur de la séparation des élèves).

Si les questions de gestion de l'hétérogénéité des élèves et d'attribution des ressources à l'école appellent le plus souvent à parler de justice scolaire (Friant, 2012, 2013 ; Monseur \& Demeuse, 2001), on retrouve clairement dans ces résultats les deux paradigmes du concept de justice sociale : la reconnaissance et la redistribution. La volonté de combattre la catégorisation des élèves faibles du système vaudois renvoie à un souci de reconnaissance sociale : le stigmate associé à la VSO étant susceptible d'induire une dévalorisation sociale des élèves (Croizet \& Leyens, 2003) perpétrée par elles/eux-mêmes, leurs enseignant-es et, dans la même logique, leurs éventuel-les employeurs/euses. L'enjeu de reconnaissance sociale est donc porté par les partisan-es d'une diminution, voire du bannissement des filières ; la suppression de la VSO poursuit un but de non-stigmatisation et de valorisation des

\footnotetext{
15 Nous justifions le choix de ce terme par son usage dans la presse vaudoise, qui traite régulièrement des débats de la réforme en empruntant au champ lexical de la guerre ; le mot " camp » est le plus utilisé, suivi des mots « guerre » et « combat » (Revaz, 2020).
} 
élèves en difficulté. L'idée de redistribution des ressources est, quant à elle, présente dans les discours des deux camps et concerne non pas les ressources matérielles, mais les ressources humaines, c'est-à-dire les enseignants et enseignantes. Du côté des partisan-es des trois voies, il s'agit de regrouper les élèves faibles pour qu'elles/ils bénéficient, ensemble, d'un enseignement et d'un accompagnement qui correspondent au niveau du groupe. Selon elles/eux, il est plus juste pour les élèves en difficulté de les isoler afin de leur offrir un enseignement adapté à leurs aptitudes et leur permettre, ainsi, d'acquérir les compétences de base. En parallèle, cette solution est aussi plus juste pour les élèves qui ne présentent pas ou peu de difficultés, car l'isolement des plus faibles leur permet d'éviter de perdre du temps et d'avancer à leur rythme. Du côté des défenseurs/euses d'un système plus hétérogène, il est question de revoir la distribution des ressources et d'accorder la même qualité d'enseignement à tous/tes les élèves afin de permettre aux plus faibles non seulement d'acquérir les compétences de base, mais d'aller au-delà de celles-ci. II s'agit, in fine, de réduire les écarts entre élèves. Ici, la justice sociale fait écho à l'égalité à la sortie de l'école : «Si tous les élèves vaudois ne peuvent prétendre accéder aux mêmes filières et atteindre les mêmes résultats, tous doivent avoir les mêmes chances de poursuivre leur parcours scolaire ou professionnel - après le secondaire I » (Revaz, 2020).

Sur le cas vaudois, les résultats des analyses révèlent l'ambivalence de l'argument de la justice sociale, exprimé notamment à travers l'idée de l'égalité des chances. Telle qu'elle apparaît dans les débats, cette ambivalence traduit la variabilité des interprétations possibles des paradigmes de reconnaissance et de redistribution. Les cadres cognitifs et normatifs y sont pour beaucoup ; chaque individu est libre de défendre sa définition et sa représentation de la justice. Les représentations « tenaces " constituent souvent un frein au changement (Palier, 2010), et c'est bien ce que l'on constate dans le canton de Vaud où les député-es en faveur de classes hétérogènes ne parviennent pas à convaincre les partisan-es des voies de modifier le système en vigueur. La dimension cognitive n'explique toutefois pas tout. La dimension institutionnalisée de la structure scolaire induit elle aussi différentes formes d'obstacles qui participent à l'ambivalence du principe de justice. Les analyses mettent en évidence le poids de l'institution scolaire vaudoise : si l'on ne compte que neuf occurrences du lemme16 " hétérog ", c'est parce que le modèle de répartition des voies est depuis longtemps institutionnalisé dans le canton (Revaz, 2020). On comprend, dès lors, que la trajectoire de la réforme est largement déterminée par ce choix passé, traduisant ainsi une forme de dépendance au sentier (Kerremans, 2001 ; Palier, 2010). C'est le résultat de l'institutionnalisation : l'institution scolaire vaudoise repose sur des évidences; des modes de faire et de penser l'école - la répartition des élèves dans des voies - qui sont devenues " naturelles » et qu'il est difficile de remettre en question (Dupriez, 2015). En somme, le cas vaudois montre que s'il est évident que la justice sociale fait écho à des cadres cognitifs et normatifs divers et variés, sa traduction en termes politiques repose aussi pour beaucoup sur

16 Les lemmes sont des formes radicales auxquelles peuvent être rajoutés des préfixes et des suffixes : " hétérogénéité »; " hétérogène(s) ». 
les réalités institutionnelles locales. Les représentations de la justice sociale et de l'égalité des chances diffèrent donc nécessairement de ce que la recherche en éducation produit sur ces questions et, par les mêmes mécanismes de production de sens, de celles des professionnel-les.

\subsection{Réponses symboliques et transformations en surface sur le terrain neuchâtelois}

La construction du sens d'une réforme se poursuit lors du passage des injonctions politiques à leur appropriation par les professionnel-les, et c'est ce que montre le cas de la réforme neuchâteloise. Elle illustre particulièrement la façon dont le sens de la réforme est contourné et bouleversé par des mécanismes de rejet de l'objectif de justice, justifiés par des représentations différentes des fonctions de l'enseignement secondaire I. Les analyses des entretiens révèlent une forme de décalage normatif entre les objectifs de justice visés par la loi et ceux que les enseignant-es attribuent à leur profession. Alors que la réforme suggère un modèle de répartition moins sélectif et davantage en harmonie avec les forces et faiblesses des élèves, la très large majorité des enseignants et enseignantes pense le secondaire I avant tout comme une étape qui doit servir à distribuer - orienter - les élèves vers les voies académiques et professionnelles qui correspondent à leurs compétences. Ainsi, regrouper les élèves de même niveau dans des classes homogènes revient à protéger les élèves qui se dirigent vers des voies académiques d'un nivellement par le bas et, en parallèle, offrir à celles/ceux qui présentent des difficultés un enseignement et un encadrement ciblés pour les aider à progresser. Dans cette perspective, les enseignant-es défendent une perspective plutôt fonctionnaliste - distributive - et méritocratique du secondaire I et ne formulent que très peu le souhait d'une amélioration de la justice sociale. Leur discours reflète une frontière subtile entre orientation et sélection, car elles/ils semblent vouloir une école qui maintient l'ordre social. Si peu contrediront la thèse selon laquelle l'école doit promouvoir une plus grande justice sociale, le discours sur la matérialisation d'un système de répartition plus équitable des élèves témoigne d'importantes contradictions. Les résultats montrent que les inégalités entre élèves sont acceptées, sous prétexte de la mission d'orientation du secondaire I au regard du niveau scolaire des élèves. Le paradigme de la reconnaissance est ainsi dominé par une aspiration méritocratique, présente également dans le cas vaudois. Dans une telle logique, un enseignement équitable, répondant au paradigme de redistribution, a peu de place. Les propos de cet enseignant sont révélateurs de cette pensée largement partagée au sujet de la finalité de l'enseignement secondaire I :

Évidemment, c'est de trouver une solution pour la fin de la scolarité, pour moi c'est le but principal. En tout cas, c'est mon but avec mes élèves. (...) Mais le but du secondaire I, c'est ça. C'est vraiment de pouvoir trouver une solution convenable en fonction du niveau de chaque élève en fait.

Lorsque les analyses portent sur les pratiques rapportées des enseignant-es et des membres des directions d'établissement, nous observons une très forte inertie entre le système 
précédent et celui qu'a introduit la réforme. Bien que le nouveau règlement soit en vigueur, les professionnel-les de l'enseignement usent de leur marge de liberté et de leur pouvoir discrétionnaire (Lipsky, 1980) pour le réinterpréter à la lumière de leurs représentations et leurs intérêts. Cette appropriation du règlement a pour conséquence de modifier la finalité prescrite du nouveau système. En effet, parmi les pratiques rapportées, nous identifions différentes stratégies de contournement du modèle de répartition élaboré dans le règlement. Si, dans ce dernier, le système d'orientation des élèves doit être souple et leur permettre d'évoluer dans des classes de niveaux d'exigences différents dans chaque discipline et de passer d'un niveau à l'autre, c'est précisément ce qu'évitent plusieurs enseignants et enseignantes. Certain-es découragent directement les élèves :

J'ai aussi des collègues qui avertissent les élèves en disant vous aurez 5.105 .20 de moyenne au semestre, mais n'imaginez pas que vous puissiez être suffisants au deuxième semestre si vous passez au niveau 2. Faites très attention, il y a un immense fossé.

D'autres opèrent auprès des parents afin que ces derniers/ères dissuadent leur(s) enfant(s) de toute volonté de passage du niveau 1 au niveau 2, passage qui traduirait une progression dans les apprentissages. II s'agit ici d'un effet de cooling out (Clark, 1960), mécanisme qui consiste, pour les enseignant-es, à inscrire les élèves dans des voies de réussites qu'elles/ils jugent réalistes. Autrement dit, le corps enseignant circonscrit les ambitions des élèves au regard de la conception qu'elles/ils ont des capacités de ces derniers/ères. Or, si la possibilité de passer d'un niveau à l'autre n'est pas saisie, les parcours continuent d'être cloisonnés. Ils le sont d'autant plus que d'autres stratégies sont mobilisées pour restreindre les réorientations progressives des élèves. Bien qu'il existe un certain nombre de disciplines à niveau, le règlement stipule que dans l'ensemble des autres disciplines, les élèves doivent être mélangé-es indépendamment de leur niveau scolaire afin de constituer des groupes hétérogènes et éviter un nouveau cloisonnement des parcours. Au sein d'un établissement scolaire, une sous-directrice confie s'arranger pour rassembler les élèves de niveau 2 en mathématiques et en sciences de la nature dans les mêmes classes, indépendamment de la discipline. Par exemple, pour les cours d'histoire et de géographie, les classes doivent regrouper des élèves de niveau 1 et de niveau 2. Dans les faits, la sous-directrice constitue des groupes homogènes soit de niveau 1, soit de niveau 2. Dans cette perspective, la volonté politique qui consistait à décloisonner l'enseignement et à réduire la sélection des élèves est loin d'être mise en œuvre. Dans ce cas précis, cette stratégie se justifie par les intérêts de l'équipe de direction d'une part, et par les représentations de ses membres d'autre part. Les intérêts sont exprimés à travers la question des désagréments occasionnés par la nouvelle structure organisationnelle de l'établissement. La " lourdeur administrative » engendrée par le nouveau système est évoquée comme un obstacle au bon fonctionnement de l'établissement : pour gagner du temps, la direction se dit obligée de contourner le règlement et d'opérer cette refiliarisation hybride. Si, à certains égards, nous pouvions penser qu'il s'agisse d'une adaptation provisoire, la sous-directrice présente cet aménagement comme pérenne. 
En définitive, le cas de la réforme neuchâteloise montre que les principes au cœur d'une réforme, tels qu'ils sont formulés par la recherche et par les acteurs/trices politiques, viennent nécessairement se heurter non seulement aux idées et aux contraintes institutionnelles auxquelles elles/ils font face, mais aussi à leurs intérêts. Ces résultats emboîtent d'ailleurs parfaitement le pas des observations réalisées par Weick (1976), Lipsky (1980) ou encore Lessard, et al. (2008a) plus récemment, qui font état d'importants découplages entre la politique et ses effets : ici, les professionnel-les adaptent certaines de leurs pratiques à la marge, afin de rendre visible la transformation désirée par la réforme, sans pour autant modifier profondément leurs pratiques ou du moins, pas à la hauteur de ce qui est attendu de leur part. Ces résultats posent d'importantes questions sur la capacité des acteurs/trices à modifier des structures en s'affranchissant de la dépendance au sentier emprunté. La réforme neuchâteloise illustre ainsi la dimension incrémentale du changement (Draelants, 2009 ; Thelen, 2003), en cela qu'elle montre la façon dont les pratiques institutionnalisées des professionnel-les résistent, sans nécessairement rester insensibles à toutes formes de modifications à moyen et long terme.

\section{DISCUSSION ET CONCLUSION : UNE « RÉINVENTION DE L'INVENTION »}

La recherche en sciences de l'éducation sur la justice sociale produit, depuis de nombreuses années, des résultats éclairant les mécanismes sous-jacents à la production des inégalités sociales à l'école. L'analyse de cette littérature rend compte, notamment, de la responsabilité de l'école dans la création de ces inégalités et met particulièrement en évidence le poids des facteurs structurels sur les écarts de compétences qui séparent les élèves issu-es de milieux socioéconomiques différents. Or, bien que la structure de l'école et l'organisation de l'enseignement répondent à des injonctions formulées dans des lois et à des pratiques appliquées sur le terrain, cela signifie que les acteurs/trices politiques et les professionnel-les de l'éducation construisent du sens autour de la justice sociale. Partant, le but de cet article était de comprendre comment ces acteurs/trices définissent et conçoivent la justice sociale. Les réponses apportées par les deux études de cas présentées montrent qu'il n'y a pas d'élaboration ou de mise en œuvre unique de réforme de justice sociale à l'école, mais des processus de réforme multiples qui produisent des politiques différentes. Cette multiplicité s'explique par divers facteurs contextuels. D'une part, les acteurs/trices appréhendent la justice sociale à l'école au travers du prisme de leur expérience concrète (Lessard \& Carpentier, 2015). Leur idée du juste à l'école et dans la société en général dépend de cette expérience, mais aussi des intérêts liés à l'exercice de leur profession et du fonctionnement institutionnel local dans lequel elles/ils s'insèrent. D'autre part, les réformes ont pour ambition de changer une réalité fortement institutionnalisée, dont les éléments constitutifs se sont imposés comme des évidences naturelles qui résistent au changement (Dupriez, 2015). 
Dans les règlements instaurés par les deux réformes, la justice et l'égalité des chances se matérialisent par une plus grande égalité de traitement qui se traduit par une réduction de la sélection des élèves et un accroissement de l'hétérogénéité des classes. Mais les analyses montrent que les pratiques sélectives sont toujours valorisées dans les représentations des député-es parlementaires et professionnel-les et qu'elles sont toujours mises en pratique par ces derniers/ères. Si l'orientation s'apparente souvent à de la sélection, c'est notamment parce que les structures homogènes sont pensées comme les plus adaptées à la diversité des compétences et des profils des élèves ; elles reproduisent la structure sociale et permettent autant de former des élites que d'accompagner les élèves en difficultés. Les cadres cognitifs et normatifs soutenus par les acteurs/trices impliqué-es influent donc très clairement sur la définition de ce qui est juste pour les élèves et, par là même, sur la structure de l'école. Les intérêts du corps enseignant comptent aussi pour beaucoup dans l'appropriation d'injonctions relatives à la justice sociale. Bien que les réformes appellent à une plus grande hétérogénéité des classes, les conditions d'enseignement produites par des groupes d'élèves de niveaux inégaux incitent les professionnel-les à contourner les exigences des règlements et à dissimuler, derrière des changements symboliques, le maintien de leurs pratiques. C'est ainsi que les membres des directions neuchâteloises constituent des classes homogènes, rejetant l'injonction de l'hétérogénéité. Enfin, nous avons vu que le caractère institutionnalisé de certains fonctionnements peut aussi peser dans la définition que les acteurs/trices politiques et professionnel-les ont de la justice sociale. La structure traditionnelle de l'école en Suisse, qui procède depuis plusieurs décennies à la séparation des élèves de niveaux différents, est fortement ancrée et détermine largement ce qui est juste pour les député-es parlementaires et les professionnel-les de l'enseignement du point de vue des apprentissages des élèves.

Dans les deux cantons, le paradigme de reconnaissance, qui réfute les « modèles sociaux de représentation » (Fraser, 2004) dans lesquels les injustices culturelles sont acceptées, peine à s'imposer dans les processus d'élaboration et de mise en œuvre des réformes, malgré des démarches en sa faveur. C'est ce que nous avons vu avec le cas vaudois, qui illustre la façon dont les principes d'hétérogénéité et d'égalité des chances sont dépassés par une forte dépendance au sentier. Si, dans le canton de Neuchâtel, la réforme parvient à modifier, dans la loi et le règlement, des modes de faire depuis longtemps institutionnalisés (la répartition des élèves dans des filières), nos résultats indiquent que l'inertie se matérialise différemment, à travers des contournements de nouvelles règles, traduisant aussi, mais sous une autre forme, une dépendance au sentier. Quant au paradigme de redistribution, qui vise à corriger les inégalités socioéconomiques liées au genre et à l'ethnie (Fraser, 2004), il est également dominé par la volonté de maintenir une élite et d'éviter le nivellement par le bas.

In fine, la définition de la justice sociale à l'école fait inévitablement l'objet d'une traduction et d'une appropriation par celles/ceux qui ont la tâche de la rendre concrète, dans la loi comme sur le terrain. Les représentations de la justice sociale sont, par conséquent, au cœur de 
l'action publique ; dans les processus d'élaboration des politiques, comme l'illustre l'étude de cas sur la réforme vaudoise et dans les processus de leur mise en œuvre, comme nous l'avons montré à travers le cas de Neuchâtel. Ainsi, et pour reprendre les termes de Dupriez (2015), l'ensemble des processus politiques constituent toujours des « réinventions de l'invention ».

\section{RÉFÉRENCES}

Avvanzino, P. (1993). Histoire de l'éducation spécialisée (1827-1970). Les arcanes du placement institutionnel. Lausanne : Éditions EESP.

Becker, R. (2013). Inégalité et justice dans l'éducation en Suisse. Revue suisse des sciences de l'éducation, 35(3), 415-423. http://dx.doi.org/10.25656/01:10301

Bourdieu, P., \& Passeron, C. (1964). Les Héritiers. Les étudiants et la culture. Paris : Minuit.

Clark, B. R. (1960). The 'Cooling-out' Function in Higher Education. American Journal of Sociology, 65(6), 569-576. https://doi.org/10.1086/222787

Coburn, C. E. (2004). Beyond Decoupling : Rethinking the Relationship between the Institutional Environment and the Classroom. Sociology of Education, 77(3), 211-244. https://doi.org/10.1177/003804070407700302

Coleman, J.-S., Campbell, E.-Q., Hobson, C.-F., McPartland, J.-M., Mood A.-M., Weinfeld, F.-D., \& York R.-L. (1966). Equality of Educational Opportunity. Washington, DC: US. Department of Health, Education and Welfare, Government Printing Office.

Croizet, J.-C., \& Leyens, J.-P. (2003). (Eds.). Mauvaises réputations. Réalités et enjeux de la stigmatisation sociale. Paris : Armand Colin.

Draelants, H. (2009). Réforme pédagogique et légitimation. Le cas d'une politique de lutte contre le redoublement. Bruxelles : Editions De Boeck Université.

Dubet, F. (2004). L'école des chances : qu'est-ce qu'une école juste ? Paris : Seuil

Dubet, F. (2014a). Inégalités et justice sociale. Paris : La Découverte.

Dubet, F. (2014b). Sociologies de l'école. In J. Beillerot, \& N. Mosconi (Eds.), Traité des sciences et des pratiques de l'éducation (pp. 65-77). Paris : Dunod.

Dumay, X., \& Dupriez, V. (2009). L'efficacité dans l'enseignement. Promesses et zones d'ombres. Bruxelles : De Boeck Supérieur.

Dupriez, V. (2015). Peut-on réformer l'école ? Approches organisationnelle et institutionnelle du changement pédagogique. Louvain-la-Neuve : De Boeck.

Dupriez, V., \& Verhoeven, M. (2006). Débat sur l'égalité à l'école. Fondements normatifs et politiques éducatives en Belgique francophone. Les Temps Modernes, 637-638-639(3), 479-501. https://doi.org/10.3917//tm.637.0479

Duru-Bellat, M., Mons, N., \& Suchaut, B. (2004). Organisation scolaire et inégalités sociales de performances. Les enseignements de l'enquête PISA. Education et Formations, 70, 123-131. https://hal.archives-ouvertes.fr/halshs-00103515

Felouzis, G. (2020). Les inégalités scolaires. Paris : Presses Universitaires de France.

Felouzis, G., \& Goastellec, G. (2015). Les inégalités scolaires en Suisse. Berne : Peter Lang. 
Fraser, N. (2004). Justice sociale, redistribution et reconnaissance. Revue du MAUSS, 23(1), 152-164. https://doi.org/10.3917/rdm.023.0152

Fraser, N. (2010). Qui compte comme sujet de justice ? la communauté des citoyens, I'humanité toute entière ou la communauté transnationale du risque ? Rue Descartes, 67(1), 50-59. https://doi.org/10.3917/rdes.067.0050

Friant, N. (2012). Vers une école plus juste : Entre description, compréhension et gestion du système. Mons : Université de Mons. https://tel.archives-ouvertes.fr/tel-00752087

Friant, N. (2013). Égalité, équité et justice en éducation. Entornos, 26(1), 137-149. https:// halshs.archives-ouvertes. fr/halshs-01389373/document

Garraud, P. (2010). 'Agenda/émergence'. In L. Boussaguet, S. Jacquot, \& P. Ravinet (Eds.), Dictionnaire des politiques publiques (pp. 58-67). Paris : Presses de Sciences Po. https:// doi.org/10.3917/scpo.bouss.2014.01.0058

Kerremans, B. (2001, 6 avril). Institutionalism, Multi-Level Governance, and the European Union's Actions in External Trade Negociations: Some Clues for Research [communication orale]. 8e workshop de I'ECPR, Grenoble, France. https://ecpr.eu/Events/Event/ PaperDetails/5427

Lessard, C., \& Carpentier, A. (2015). Politiques éducatives. La mise en œuvre. Paris : Presses Universitaires de France.

Lessard, C., Desjardins, P.-D., Schwimmer, M., \& Anne, A. (2008a). Les politiques et les pratiques en éducation : un couplage problématique. Une perspective anglo-américaine. Carrefours de l'éducation, 25(1), 155-194. https://doi.org/10.3917/cdle.025.0155

Lessard, C., Kamanzi, P. C., \& Larochelle, M. (2008b). La perception des politiques éducatives chez les directions d'école et les enseignant-es canadiens : l'influence de l'idéologie professionnelle. Les nouvelles politiques d'éducation et de formation, 40(1), 93-118. https://doi.org/10.7202/019474ar

Lipsky, M. (1980). Street-Level bureaucracy: dilemmas of the individual in public services. New York, NY: Russell Sage Foundation.

Lynch, K., \& Payet, J.-P. (2011). L'égalité en éducation : redistribution, reconnaissance, représentation et relations affectives. Éducation et sociétés, 27(1), 5-22. https://doi.org/ 10.3917/es.027.0005

Meuret, D. (1999). La justice du système éducatif. Bruxelles : De Boeck.

Mons, N. (2007). Les nouvelles politiques éducatives. La France fait-elle les bons choix ? Paris : Presses Universitaires de France.

Monseur, C., \& Crahay, M. (2008). Composition académique et sociale des établissements, efficacité et inégalités scolaires : une comparaison internationale. Revue française de pédagogie, 164, 55-66. https://doi.org/10.4000/rfp.2128

Monseur, C., \& Demeuse, M. (2001). Gérer l'hétérogénéité des élèves. Méthodes de regroupement des élèves dans l'enseignement obligatoire. Cahiers du Service de Pédagogie expérimentale. Liège : Université de Liège. 
Muller, P. (2000). L'analyse cognitive des politiques publiques : vers une sociologie politique de l'action publique. Revue française de science politique, 50(2), 189-208. https://doi.org/ 10.3406/rfsp.2000.395464

Muller, P. \& Surel, Y. (1998). L'analyse des politiques publiques. Paris : Éditions Montchrestien.

Paindorge, M., Kerneis, J., \& Fontanieu, V. (2015). Analyse de données textuelles informatisée : l'articulation de trois méthodologies, avantages et limites. Nouvelles perspectives en sciences sociales, 1(11), 65-92. https://doi.org/10.7202/1035933ar

Palier, B. (2010). Path dependence (Dépendance au chemin emprunté). In L. Boussaguet, S. Jacquot, \& P. Ravinet (Eds.), Dictionnaire des politiques publiques (pp. 411-419). Paris : Presses de Sciences Po. https://doi.org/10.3917/scpo.bouss.2014.01.0411

Palier, B., \& Surel, Y. (2005). Les 'trois l' et l'analyse de l'Etat en action. Revue française de science politique, 55, 7-32. https://doi.org/10.3917/rfsp.551.0007

Revaz, S. (2020). Réformer l'école dans un contexte de démocratie directe: regards croisés sur trois réformes de l'enseignement secondaire obligatoire en Suisse romande. Thèse de doctorat en sciences de l'éducation, Université de Genève. https://doi.org/10.13097/ archive-ouverte/unige:137426

Surel, Y. (2000). The Role of Cognitive and Normative Frames in Policy-Making. Journal of European Public Policy, 7(4), 495-512. https://doi.org/10.1080/13501760050165334

Thelen, K. (2003). Comment les institutions évoluent : perspectives de l'analyse comparative historique. L'Année de la régulation, 7, 13-43.

https://doi.org/10.3917/scpo.colle.2003.01.0013

Van der Maren, J.-M. (1996). Méthodes de recherche pour l'éducation. Bruxelles : De Boeck. Weick, K. (1976). Educational Organizations as Loosely Coupled Systems. Administrative Science Quarterly, 21(1), 1-19. https://doi.org/10.2307/2391875

Open Access Publications - Bibliothèque de l'Université de Genève Creative Commons Licence 4.0

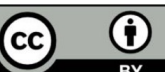

